\title{
Association of serum Cyr61 levels with peripheral arterial disease in subjects with type 2 diabetes
}

\author{
Bin Feng ${ }^{1}$, Guidong Xu' ${ }^{2}$, Kangyun Sun ${ }^{2}$, Kaipeng Duan ${ }^{3}$, Bimin Shi ${ }^{1}$ and Nannan Zhang ${ }^{2 *}$
}

\begin{abstract}
Background: The prevalence of peripheral artery disease (PAD) is obviously increased in patients with diabetes. Existing evidence shows that cysteine-rich angiogenic inducer 61 (Cyr61), a 40-kD secreted protein, plays important roles in regulating cellular physiological processes. Recent studies have demonstrated a significant correlation between serum Cyr61 and atherosclerosis. However, the relationship between Cyr61 levels and PAD in patients with type 2 diabetes (T2DM) remains obscure.
\end{abstract}

Methods: Data from a total of 306 subjects with T2DM were cross-sectionally analysed. The extent of PAD was determined by using the Fontaine classification, which defines four stages. We measured serum Cyr61 concentrations by ELISA in subjects with and without PAD at Fontaine's stage II, III, or IV. Logistic regression models were used to examine the independent association of Cyr61 with PAD.

Results: Out of the 306 subjects enrolled, 150 were free from PAD, while 156 had clinically significant PAD. In subjects with PAD, the prevalences of Fontaine classification stages II, III and IV were 48.7\%, 32.1\%, and 19.2\%, respectively. Patients with more advanced PAD had significantly higher Cyr61 ( $P$ for trend $<0.001$ ). The prevalence of PAD on the basis of severity increased with increasing Cyr61 quartiles (all $P$ values for trends $<0.001$ ), and the severity of PAD was positively correlated with Cyr61 quartiles $(r=0.227, P=0.006)$. The association of Cyr61 levels with PAD remained after adjusting for major risk factors in a logistic regression analysis.

Conclusions: Our results demonstrated that Cyr61 was significantly increased in PAD patients with T2DM and that Cyr61 levels were positively associated with disease severity. Cyr61 could be a promising biomarker and further studies are needed to assess its clinical utility.

Keywords: Cyr61, Type 2 diabetes, Peripheral artery disease, Atherosclerosis

\section{Background}

Peripheral artery disease (PAD) is a common manifestation of atherosclerotic disease, which is related to considerable disability and mortality. Currently, approximately 202 million people worldwide are

\footnotetext{
*Correspondence: zhangnannan0520@126.com

2 Department of Cardiology, The Affiliated Suzhou Hospital of Nanjing

Medical University, Jiangsu Province, 242 Guangji Road, Suzhou 215008, PR China

Full list of author information is available at the end of the article
}

suffering from lower extremity arterial disease, giving rise to a major public health problem and a heavy economic burden [1,2]. Type 2 diabetes (T2DM) is one of the major risk factors for atherosclerosis, and the prevalence of PAD increases with the prevalence of T2DM. Moreover, compared with nondiabetic individuals, patients with diabetes have a poorer prognosis for PAD $[1,2]$. Therefore, early diagnosis and intervention of PAD in patients with diabetes are essential to reduce the risk of major adverse limb events (MALEs) [3]. At present, various international guidelines recommend 
the ankle brachial index (ABI) as the preferred screening tool for PAD in diabetic individuals [4]. Due to the low sensitivity of the ABI for the detection of earlystage PAD, there is an urgent need to find novel biomarkers that can identify PAD among patients with diabetes in the initial stage.

Previous studies have found that some members of the cellular communication network $(\mathrm{CCN})$ family are highly expressed in atherosclerotic plaques, contributing to the development of cardiovascular and cerebrovascular diseases and peripheral arterial diseases $[5,6]$. Cysteine-rich angiogenic inducer 61 (Cyr61), belonging to the CCN family, is a $40 \mathrm{kD}$ secreted extracellular matrix (ECM)- related signalling protein that can regulate cell proliferation, adhesion, differentiation and extracellular matrix production [7-9]. Cyr61 is maintained at a low level under normal conditions, but is usually elevated in various states of disease, such as colitis, rheumatoid arthritis, Graves' orbitopathy, diabetic retinopathy and atherosclerosis [10-15]. Downregulation of Cyr61 expression in carotid balloon injury rats can mitigate the proliferation of vascular smooth muscle cells, thereby attenuating vascular intimal hyperplasia [16]. Moreover, inhibition of the Cyr61 signalling pathway contributes to the reduction in vascular smooth muscle cell senescence in human coronary artery smooth muscle cells [17]. Additionally, recent studies demonstrated that Cyr61 levels were independently associated with 30-day mortality in patients with acute heart failure (AHF) and coronary heart disease (CAD) [18], and could be a potential marker of myocardial ischemic injury and prognosis in patients with acute coronary syndrome (ACS) $[9,19]$. To date, however, the link between circulating Cyr61 and PAD in patients with diabetes has not yet been established.

Since numerous studies have demonstrated links between Cyr61 and various aspects of atherosclerosis $[11,20]$ and diabetic microvascular complications [10], it is reasonable to hypothesize a correlation between Cyr61 and PAD in the diabetic condition. Therefore, we sought to investigate the potential role of Cyr61 as a marker of endothelial dysfunction and PAD in patients with T2DM.

\section{Methods \\ Study population and design}

We performed a cross-sectional study that was approved by the Ethics Committee of the First Affiliated Hospital of Soochow University and the Affiliated Suzhou Hospital of Nanjing Medical University in accordance with the principles of the Helsinki Declaration. Written informed consent was obtained from each participant. PASS 15.0 software (NCSS, LLC) was used to calculate the sample size. Two-sided intervals and $95 \%$ confidence were required. The expected standard deviation of serum Cyr61 was $13.0 \mathrm{pg} / \mathrm{ml}$, and $1.5 \mathrm{pg} / \mathrm{ml}$ margin error is allowed. The calculated sample size is $\mathrm{N}=289$ for this study.

Individuals with T2DM were consecutively recruited from the Department of Endocrinology and Metabolism of the First Affiliated Hospital of Soochow University and the Department of Cardiology of the Affiliated Suzhou Hospital of Nanjing Medical University, Jiangsu, China, from 1 October 2018 to 31 March 2020. The diagnosis of diabetes is based on the 1999 World Health Organization (WHO) criteria. Inclusion criteria were age $\geq 18$ years with the presence of T2DM. Exclusion criteria included renal failure with estimated glomerular filtration rate (eGFR) $<30 \mathrm{ml} / \mathrm{min}$; acute infectious disease at the time of evaluation; a history of malignancy, mental disorders, autoimmune diseases, or severe heart or liver dysfunction; and history of solid or hematological neoplasia or active neoplasia. For each participant in the study, anklebrachial index (ABI) measurement was performed, and according to the judgement of the attending physician, both lower limbs were further assessed using arterial Doppler ultrasound-enhanced ultrasonography, computed tomography (CT) angiography, or lower limb angiography. The participants were considered to have PAD if: they had a previous history of lower limb percutaneous transluminal angioplasty, with or without stenting; or they met at least one instrumental and one clinical criterion listed in Table 1. Subjects with an ABI $>0.90$ and no

Table 1 Criteria for PAD definition in patients without a history of lower limb amputation, PTA or bypass surgery

\begin{tabular}{ll}
\hline Clinical Criteria & Instrumental criteria \\
\hline Presence of Intermittent claudication & $\mathrm{ABI}<0.90$ \\
Resting pain & $\mathrm{TCPO}_{2}<30 \mathrm{mmHg}$ \\
Non-healing distal ulcer & $\begin{array}{l}\text { Ultrasound or angiography found atherosclerosis with stenosis, with a reduction at } \\
\text { Gangrene }\end{array}$ \\
least $50 \%$ of the lumen diameter, consistent with clinical symptoms \\
Ultrasonographic finding of post-stenotic blood flow profile, consistent with symptoms
\end{tabular}

PTA, percutaneous transluminal coronary angioplasty 
clinical manifestations of PAD (intermittent claudication, resting pain, non-healing distal ulcer, or gangrene) were deemed to be without PAD and did not undergo further testing.

The extent of PAD was determined by using the Fontaine classification, which defines four stages: stage I, asymptomatic; stage II, intermittent claudication; stage III, resting pain; and stage IV, ischemic ulcers or gangrene [21].

\section{Anthropometric and biochemical measurements}

Anthropometric measurements included height, weight and blood pressure. Body mass index (BMI) was defined as the weight in kilograms divided by their height (in meters) squared. From each subject, a 10-ml fasting peripheral blood sample was collected and centrifuged for $5 \mathrm{~min}$ at a rotating speed of 3,000. The serum was immediately frozen at $-80{ }^{\circ} \mathrm{C}$. Total cholesterol (TC), triglycerides (TG), high-density lipoprotein cholesterol (HDL-C), and low-density lipoprotein cholesterol (LDLC) were measured by applying standard enzymatic methods using a biochemical analyser (HITACHI 7450, Tokyo, Japan). Fasting glucose levels were determined by the glucose oxidase method. $\mathrm{HbA}_{1 \mathrm{c}}$ was assayed by using highperformance liquid chromatography with a VARIANT II Hemoglobin Testing System (Bio-Rad Laboratories, Hercules, CA). eGFR was calculated using the simplified Modification of Diet in Renal Disease (MDRD) formula. Serum Cyr61 was determined by a commercially available ELISA kit (R\&D Systems, Minneapolis, MN) according to the manufacturer's instructions. For each subject, the serum sample was measured twice, and the results were averaged. The hospital's clinical laboratory center implements internal and external quality control procedures. The intra- and inter-assay coefficients of variation are presented in Additional file 1: Table S1.

\section{Statistical analysis}

Demographic and clinical data of the groups were compared using the $x^{2}$ test and t-test. For non-normally distributed variables, a logarithmic transformation was carried out before further analysis. The trends of continuous variables across the various groups were assessed with the use of linear polynomial contrasts in ANOVA for normally distributed variables and the JonckheereTerpstra test for non-normally distributed data. The Cochran-Armitage trend test was used to examine trends of rates across groups. Status by severity of PAD was treated as an ordinal categorical variable $(0=$ non-PAD, $1=$ Fontaine stage II, $2=$ Fontaine stage III, and $3=$ Fontaine stage IV). Binary logistic regression analysis was performed to investigate the effect of serum Cyr61 on the risk of PAD. The area under the receiver operating characteristic (ROC) curve was calculated to test the discrimination of PAD. Graphs were created using Prism 8.0 (GraphPad Software), and statistical analysis was performed with GraphPad Prism. A $P$ value $<0.05$ was considered to be statistically significant.

\section{Results}

The clinical characteristics of the subjects are shown in Table 2. Among the 306 subjects with diabetes enrolled in the study, 156 were included as PAD, and 150 as no PAD. The mean \pm SD age of the enrolled participants was $61.2 \pm 11.4$ years; they had a mean \pm SD diabetes duration of $8.6 \pm 5.6$ years, fasting glucose of $7.4 \pm 1.6 \mathrm{mmol} / \mathrm{L}$ and $\mathrm{HbA}_{1 \mathrm{c}}$ of $8.8 \pm 1.7 \%$. There were no significant differences between groups regarding BMI $(P=0.640)$, smoking status $(P=0.953)$, blood pressure $(P=0.344)$, TC $(P=0.172)$, LDL-C $(P=0.557)$ or TG $(\mathrm{P}=0.692)$. In individuals with PAD, the prevalences of Fontaine classification stage II, III and IV were $48.7 \%, 32.1 \%$, and $19.2 \%$, respectively. Serum Cyr61 concentrations differed significantly across the various groups and increased progressively across the severity of PAD $(P$ for trend $<0.001)$. Individuals with more severe PAD had a longer diabetes duration; higher HDL-C, fasting glucose and $\mathrm{HbA}_{1 \mathrm{c}}$ levels; and lower eGFR. They were more likely to be treated with insulin and had a higher propensity to receive renin-angiotensin-aldosterone system (RASS) inhibitors (Table 2).

Next, all of the participants were stratified according to quartiles of serum Cyr61 level (quartile 1 [Q1]: $\leq 169.7 \mathrm{pg} / \mathrm{ml}$; quartile 2 [Q2]: 169.7-206.6 pg/ $\mathrm{ml}$; quartile 3 [Q3]: $206.6-282.3 \mathrm{pg} / \mathrm{ml}$; and quartile 4 [Q4]: $\geq 282.3 \mathrm{pg} / \mathrm{ml}$ ). As shown in Fig. 1, individuals with a longer diabetes duration have a higher serum Cyr61 concentration. Levels of $\mathrm{HbA}_{1 \mathrm{c}}$ and LDL-C were increased, meanwhile, levels of HDL-C and eGFR were significantly decreased in subjects of the higher Cyr61 quartile. In general, the prevalence of PAD by severity increased with ascending quartiles of Cyr61 (all $P$ values for trends $<0.001$ ) (Fig. 2). A significant association between the Cyr61 quartile and the severity of PAD was observed after Spearman correlation analysis $(r=0.227$; $P=0.006$ ). More detailed characteristics are presented in Additional file 2: Table S2.

Logistic regression analysis revealed that serum Cyr61 was significantly associated with the risk of PAD in both the crude and adjusted models including age, sex, BMI, diabetes duration, fasting glucose, hypertension, TG, HDL, LDL, HbA1c, eGFR, smoking status, use of RAAS inhibitors, statins and fibrates as covariates (all $P$ values $<0.005$, Table 3 ).

Receiver-operator characteristic (ROC) curves were generated to evaluate the discriminative capability of 
Table 2 Demographic and clinical data of subjects with diabetes by the presence and severity of PAD

\begin{tabular}{|c|c|c|c|c|c|c|}
\hline \multirow[t]{2}{*}{ Variables } & \multirow{2}{*}{$\begin{array}{l}\text { All subjects } \\
(n=306)\end{array}$} & \multirow{2}{*}{$\begin{array}{l}\text { No PAD } \\
(n=150)\end{array}$} & \multicolumn{3}{|c|}{ Fontaine classification } & \multirow[t]{2}{*}{$P$ value for trenc } \\
\hline & & & II $(n=76)$ & III $(n=50)$ & IV $(n=30)$ & \\
\hline Male & 52.9 & 45.3 & 47.4 & 44.0 & 53.3 & 0.606 \\
\hline Age (years) & $61.2 \pm 11.4$ & $60.4 \pm 12.0$ & $59.2 \pm 11.0$ & $61.2 \pm 11.0$ & $62.6 \pm 10.8$ & 0.233 \\
\hline BMI $\left(\mathrm{kg} / \mathrm{m}^{2}\right)$ & $25.2 \pm 3.3$ & $25.1 \pm 3.4$ & $25.1 \pm 3.4$ & $25.0 \pm 3.5$ & $24.8 \pm 3.3$ & 0.640 \\
\hline Current smoker & 26.1 & 25.3 & 28.9 & 24.0 & 26.7 & 0.953 \\
\hline Hypertension & 51.0 & 48.0 & 52.6 & 56.0 & 53.3 & 0.344 \\
\hline Diabetes duration (years) & $8.6 \pm 5.6$ & $7.3 \pm 6.1$ & $9.6 \pm 6.4$ & $10.7 \pm 6.0$ & $12.8 \pm 5.8$ & $<0.001$ \\
\hline Total cholesterol (mmol/L) & $4.5 \pm 1.3$ & $4.6 \pm 1.3$ & $4.5 \pm 1.3$ & $4.7 \pm 1.3$ & $4.9 \pm 1.1$ & 0.172 \\
\hline $\mathrm{TG}(\mathrm{mmol} / \mathrm{L})$ & $1.8 \pm 1.5$ & $1.9 \pm 1.7$ & $1.8 \pm 1.5$ & $1.7 \pm 1.4$ & $1.8 \pm 1.7$ & 0.692 \\
\hline $\mathrm{HDL}-\mathrm{C}(\mathrm{mmol} / \mathrm{L})$ & $1.2 \pm 0.4$ & $1.1 \pm 0.4$ & $1.2 \pm 0.4$ & $1.2 \pm 0.4$ & $1.3 \pm 0.3$ & 0.015 \\
\hline LDL-C (mmol/L) & $3.1 \pm 0.7$ & $3.1 \pm 0.9$ & $3.1 \pm 0.7$ & $3.1 \pm 0.7$ & $3.2 \pm 0.8$ & 0.557 \\
\hline $\mathrm{HbA}_{1 \mathrm{c}}(\%)$ & $8.8 \pm 1.7$ & $8.2 \pm 1.5$ & $8.9 \pm 1.6$ & $8.9 \pm 1.5$ & $9.1 \pm 1.7$ & 0.006 \\
\hline Fasting glucose (mmol/L) & $7.4 \pm 1.6$ & $7.1 \pm 1.5$ & $7.9 \pm 1.7$ & $7.7 \pm 1.5$ & $8.1 \pm 1.5$ & 0.004 \\
\hline $\mathrm{eGFR}\left(\mathrm{ml} / \mathrm{min} / 1.73 \mathrm{~m}^{2}\right)$ & $79.4 \pm 8.4$ & $82.3 \pm 8.7$ & $79.5 \pm 8.9$ & $78.5 \pm 8.8$ & $76.5 \pm 8.4$ & $<0.001$ \\
\hline Serum Cyr61 (pg/ml) & $219.1 \pm 13.5$ & $195.4 \pm 11.9$ & $221.0 \pm 14.4$ & $247.6 \pm 11.8$ & $275.5 \pm 12.3$ & $<0.001$ \\
\hline \multicolumn{7}{|l|}{ Use antidiabetes agents } \\
\hline Oral drugs & 58.2 & 61.3 & 60.5 & 48.0 & 55.3 & 0.225 \\
\hline Insulin & 64.7 & 54.7 & 68.4 & 76.0 & 86.7 & $<0.001$ \\
\hline \multicolumn{7}{|l|}{ Use antihypertension agents } \\
\hline$\beta$-Blockers & 13.1 & 10.7 & 13.2 & 16.0 & 20.0 & 0.123 \\
\hline Calcium-channel blockers & 24.2 & 24.0 & 23.7 & 24.0 & 26.7 & 0.826 \\
\hline RAAS inhibitors & 47.1 & 40.0 & 47.4 & 56.0 & 66.7 & 0.003 \\
\hline Diuretics & 6.5 & 5.3 & 7.9 & 8.0 & 6.7 & 0.551 \\
\hline \multicolumn{7}{|l|}{ Use lipid-lowering agents } \\
\hline Statins & 24.8 & 3221.3 & 2026.3 & 1428.0 & 1033.3 & 0.119 \\
\hline Fibrates & 7.2 & 85.3 & 810.5 & 48.0 & 26.7 & 0.530 \\
\hline
\end{tabular}

Data are the mean $\pm S D$ or percentage unless otherwise indicated

$B M I$ body mass index, RAAS renin-angiotensin-aldosterone system

Cyr61 for determination of PAD and to compare it with the $\mathrm{ABI}$. In all subjects with diabetes, the area under the curve (AUC) for Cyr61 was significantly greater than that for the ABI (Fig. 3). The best cut-off value of Cyr61 for PAD detection in the present study was $>233.7 \mathrm{ng} /$ $\mathrm{ml}$ (sensitivity $82.7 \%$, specificity $72.3 \%$ ).

\section{Discussion}

Peripheral arterial disease usually seriously affects the daily quality of life of patients and imposes huge personal and societal healthcare burdens [22, 23]. To the best of our knowledge, this is the first study to evaluate the association between serum Cyr61 levels and the risk of PAD. However, due to the cross-sectional nature of the study design, it is impossible to determine the causality between Cyr61 and PAD. Thus, we have to be careful about interpreting the associations concluded from this survey.

\section{Potential biomarkers for diabetes with PAD}

Considering the relevance of the disease, effective earlier screening and diagnosis of PAD for patients with diabetes has become increasingly important. However, there is still a lack of effective biomarkers with high sensitivity and specificity for early diagnosis, and often a patient consults the specialist when the disease is already significantly worsened. Several potential biomarkers were found to identify PAD in patients with diabetes. For instance, serum high mobility group box (HMGB) 1, fibroblast growth factor (FGF) 23 and osteopontin (OPN) have been correlated with the presence of PAD [24, 25]. In addition, thrombospondin-4 (TSP-4) levels were significantly increased with PAD severity in patients with concomitant diabetes and could be a novel marker of atherosclerotic activity [26]. Recently, the association between circulating serum growth differentiation factor (GDF) 15 and lower extremity atherosclerotic disease (LEAD) has been investigated in Chinese diabetes 


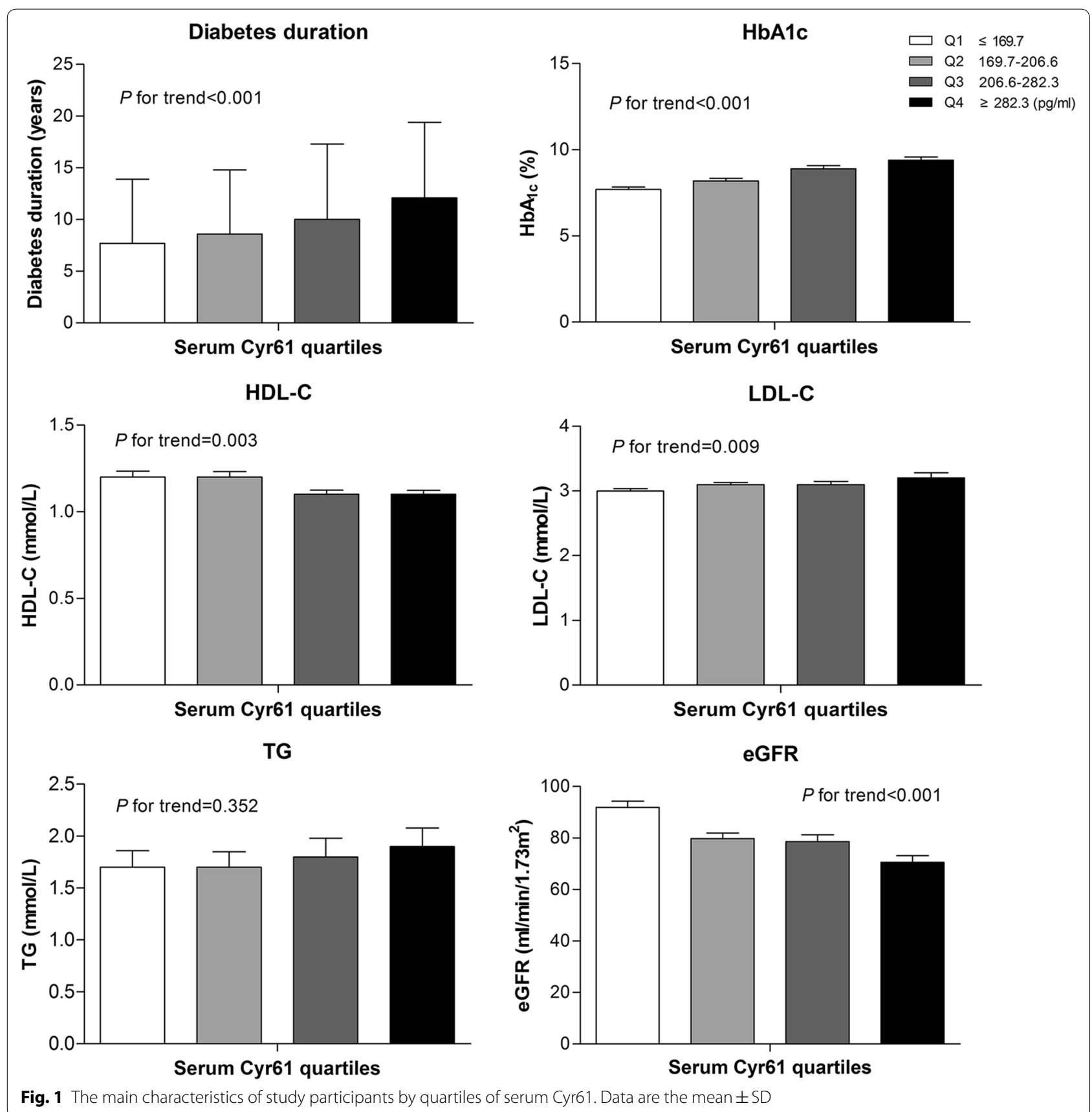

subjects [27]. Furthermore, Hayashi et al. reported a method for accurate skin temperature measurement using noncontact, handheld infrared skin thermometer, which could serve as a new, cost-effective screening strategy for earlier diagnosis of PAD [28]. Very recently, Biscetti and colleagues reported that elevated plasma sortilin is significantly and independently associated with the presence of lower limb PAD in statin-free patients with diabetes, while omentin-1 is reduced in the same population. Both of them could be promising markers for clinically significant atherosclerosis in the lower limbs $[29,30]$.

\section{Cyr61 and peripheral vascular injuries}

Cyr61 is a multifunctional matricellular protein that plays essential roles in regulating inflammation, wound healing and fibrogenesis in adults. Aberrant Cyr61 expression is associated with numerous pathologies, including diseases 


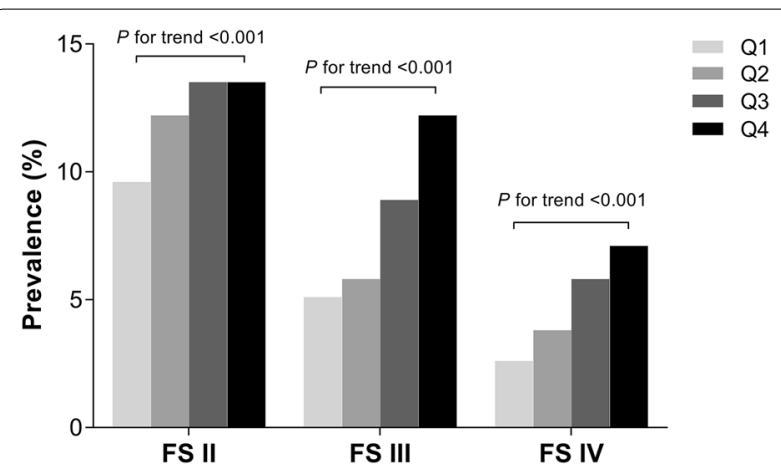

Fig. 2 Prevalence of PAD by severity as a function of Cyr61 quartile. Cyr61 quartile: Q1: 169.7 pg/ml, Q2: 169.7-206.6 pg/ml, Q3: 206.6-282.3 pg/ml, Q4: $\geq 282.3 \mathrm{pg} / \mathrm{ml}$. FS Fontaine Stage

Table 3 Association of serum Cyr61 with PAD by logistic regression analyses.

\begin{tabular}{lllr}
\hline Modes & OR & 95\% confidence interval & $P$ value \\
\hline Crude & 0.915 & $0.854-0.949$ & $<0.001$ \\
Mode1 & 0.923 & $0.867-0.964$ & 0.005 \\
Mode2 & 0.936 & $0.872-0.981$ & 0.008 \\
Mode3 & 0.947 & $0.879-0.993$ & 0.048 \\
\hline
\end{tabular}

Model 1 is adjusted for age, sex and BMI; Model 2 includes all variables in Model 1 plus diabetes duration, fasting glucose, hypertension, TG, HDL-C and LDL-C; Model 3 includes all variables in Model 2 plus HbA1C, eGFR, smoking status, use of RAAS inhibitors, statins and fibrates

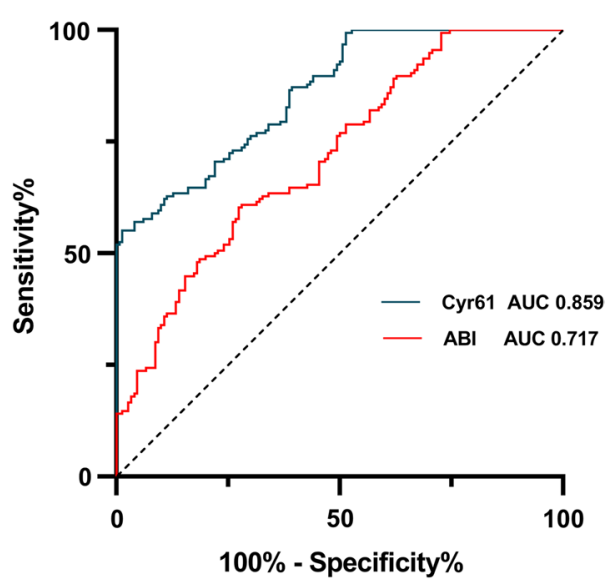

Fig. 3 Receiver operating characteristic (ROC) curves for Cyr61 and $A B I$ in 306 subjects with diabetes. The curves show discriminative capability for determination of PAD between Cyr61 and ABI measurements. $R O C$ receiver operating characteristic, $A \cup C$ area under the curve

associated with autoimmune, metabolism and chronic inflammation [31]. Furthermore, regulation of the Cyr61 level in the circulation and vital organs has also been observed during experimentally induced sepsis [32, 33]. In terms of peripheral vascular disease, previous studies have demonstrated that Cyr61 is overexpressed in vascular smooth muscle cells of atherosclerotic lesions both in humans and in animal models $[20,34,35]$ and could stimulate adhesion of vascular smooth muscle cells in a dose-dependent manner [11]. Interestingly, plasminogen (Plg)-mediated Cyr61 cleavage and activation promoted endothelial cell migration and neovascularization in vitro and in vivo. Targeting Plg/Cyr61 may offer exciting therapeutic opportunities for strengthening mesenchymal stem cells therapy in ischemic diseases [36]. Because the concept that inflammation participates pivotally in the pathogenesis of atherosclerosis has gained considerable attention [37], we speculated that chronic low-grade inflammation, together with the increased Cyr61 concentration contribute, at least in part, to the occurrence of atherosclerotic lesions of the lower limbs in patients with diabetes. In the present study, we found that serum Cyr61 levels increase according to severity of PAD, as if there were a dose-dependent relationship. Furthermore, results of ROC curve analysis show that Cyr61 concentrations can effectively identify the presence of PAD in subjects with diabetes, and the diagnostic sensitivity and specificity are better than the ABI. Thus, serum Cyr61 may be a promising biomarker for early diagnosis and effective follow-up of PAD in patients with diabetes.

It is not possible to definitively clarify whether Cyr61 levels are a cause or an effect of PAD, or even both, through a vicious cycle. In fact, earlier studies on Cyr61 highlighted its causative roles in intima proliferation. For instance, negative regulation of the Cyr61 gene led to the inhibition of vascular smooth muscle cell proliferation and neointimal hyperplasia [11]. Matsumae and colleagues also demonstrated that knockdown of Cyr61 significantly suppressed neointimal hyperplasia in a rat carotid artery balloon injury model [16]. Thus, targeting circulating Cyr61 may provide a novel strategy for the prevention of peripheral arterial disease.

\section{Limitations}

The study has several major limitations that should be noted. First, due to the cross sectional design of the study, we could not establish a causal relationship between serum Cyr61 and the development of PAD. Nevertheless, our results support the hypothesis that Cyr61 is important in peripheral atherosclerosis physiopathology. Second, the cross-sectional measurements may not represent the participant's stable level of serum Cyr61. Therefore, the results of this study should be interpreted with caution. Third, the enrolled individuals were hospitalized patients with diabetes in the Suzhou area (Jiangsu, China), and the generalizability 
of the results to other diabetic populations needs to be tested. A further limitation is that we need prospective data to confirm whether higher Cyr61 levels may suffice as an effective biomarker for PAD in patients with diabetes. Last, because the enrolment of participants was restricted to patients with T2DM with higher susceptibility for developing PAD, selection bias of the study was an inevitable and non-negligible issue. In addition, we did not use healthy controls to determine the normal level of Cyr61. In fact, there is still a lack of studies which focused on differences of Cyr61 levels among health populations due to aging, ethnic variation, genetic background, and nutritional status. We believe that our results can provide a valuable reference for future large-scale studies.

\section{Conclusion}

In summary, we provided evidence that circulating Cyr61 levels are significantly correlated with the disease severity of PAD in the diabetes population. Although our findings underscore the value of Cyr61 as a biomarker, more prospective studies and clinical trials are warranted to better elucidate the role of Cyr61 in the onset and progression of PAD in T2DM patients. In addition, basic research is urgently needed to explore the underlying mechanisms of Cyr61 regulation under PAD conditions.

\section{Supplementary information}

Supplementary information accompanies this paper at https://doi. org/10.1186/s12933-020-01171-9.

Additional file 1: Table S1. The assay coefficients of variation for measuring biochemical parameters.

Additional file 2: Table S2. Characteristics of study participants by quartiles of serum Cyr61. Data are the mean \pm SD or percentage unless otherwise indicated. BMI body mass index; RAAS renin-angiotensinaldosterone system.

\section{Abbreviations}

PAD: Peripheral artery disease; T2DM: Type 2 diabetes; MALEs: Major adverse limb events; CCN: Cellular communication network; AHF: Acute heart failure; CAD: Coronary heart disease; ACS: Acute coronary syndrome; eGFR: Estimated glomerular filtration rate; $\mathrm{ABl}$ : Ankle-brachial index; CT: Computed tomography; BMI: Body mass index; TC: Total cholesterol; TG: Triglyceride; HDL-C: High-density lipoprotein cholesterol; LDL-C: Low-density lipoprotein cholesterol; RAAS: Renin-angiotensin-aldosterone system; ROC: Receiver operating characteristic; AUC: Area under the curve; HMGB: High mobility group box; FGF: Fibroblast growth factor; OPN: Osteopontin; Plg: Plasminogen; TSP-4: Thrombospondin-4; GDF: Growth differentiation factor; LEAD: Lower extremity atherosclerotic disease.

\section{Acknowledgements}

Not applicable.

\section{Authors' contributions}

BF conceived the study, NNZ participated in its design and coordination. BF wrote the manuscript, and GDX, KYS, KPD and BMS reviewed it. All authors read and approved the final manuscript.

\section{Funding}

This work was supported by grants from the National Natural Science Foundation of China $(81800714,81700788)$ and the Project of Suzhou Science and

Technology Plan (SYS2019094).

\section{Availability of data and materials}

The datasets used and/or analysed for this study are available from the corresponding author upon reasonable request.

\section{Ethics approval and consent to participate}

The study was approved by the local ethics committees and adhered to the principles of the Declaration of Helsinki.All patients agreed to participate in the study and gave informed consent.

\section{Consent for publication}

Not applicable.

\section{Competing interests}

The authors declare that they have no competing interests.

\section{Author details}

${ }^{1}$ Department of Endocrinology and Metabolism, The First Affiliated Hospital of Soochow University, Jiangsu Province, Suzhou 215006, P.R. China. ${ }^{2}$ Department of Cardiology, The Affiliated Suzhou Hospital of Nanjing Medical

University, Jiangsu Province, 242 Guangji Road, Suzhou 215008, PR China.

${ }^{3}$ Department of General Surgery, The First Affiliated Hospital of Soochow University, Jiangsu Province, Suzhou 215006, P.R. China.

Received: 17 Auqust 2020 Accepted: 15 November 2020

Published online: 22 November 2020

\section{References}

1. Malyar N, Fürstenberg T, Wellmann J, Meyborg M, Lüders F, Gebauer K, Bunzemeier $\mathrm{H}$, Roeder N, Reinecke $\mathrm{H}$. Recent trends in morbidity and in-hospital outcomes of in-patients with peripheral arterial disease: a nationwide population-based analysis. Eur Heart J. 2013;34(34):2706-14.

2. Déruaz-Luyet A, Raabe C, Garry EM, Brodovicz KG, Lavery LA. Incidence of lower extremity amputations among patients with type 1 and type 2 diabetes in the United States from 2010 to 2014. Diabetes Obes Metab. 2020;22(7):1132-40.

3. Nativel M, Potier L, Alexandre L, Baillet-Blanco L, Ducasse E, Velho G, Marre M, Roussel R, Rigalleau V, Mohammedi K. Lower extremity arterial disease in patients with diabetes: a contemporary narrative review. Cardiovasc Diabetol. 2018;17(1):138.

4. Association AD. 1. Improving care and promoting health in populations: standards of medical care in diabetes-2020. Diabetes Care. 2020;43(Suppl 1):s7-s13.

5. Leeuwis JW, Nguyen TQ, Theunissen MG, Peeters W, Goldschmeding R, Pasterkamp G, Vink A. Connective tissue growth factor is associated with a stable atherosclerotic plaque phenotype and is involved in plaque stabilization after stroke. Stroke. 2010;41(12):2979-81.

6. Yao Y, Li B, Fu C, Teng G, Ma G, Liu N. Anti-connective tissue growth factor detects and reduces plaque inflammation in early-stage carotid atherosclerotic lesions. Nanomedicine. 2017;13(8):2385-94.

7. Hinkel R, Trenkwalder T, Petersen B, Husada W, Gesenhues F, Lee S, Hannappel E, Bock-Marquette I, Theisen D, Leitner L, et al. MRTF-A controls vessel growth and maturation by increasing the expression of CCN1 and CCN2. Nat Commun. 2014;5:3970.

8. Krupska I, Bruford EA, Chaqour B. Eyeing the Cyr61/CTGF/NOV (CCN) group of genes in development and diseases: highlights of their structural likenesses and functional dissimilarities. Hum Genomics. 2015;9:24.

9. Klingenberg R, Aghlmandi S, Liebetrau C, Raber L, Gencer B, Nanchen D, Carballo D, Akhmedov A, Montecucco F, Zoller S, et al. Cysteine-rich angiogenic inducer 61 (Cyr61): a novel soluble biomarker of acute myocardial injury improves risk stratification after acute coronary syndromes. Eur Heart J. 2017;38(47):3493-502.

10. Hughes JM, Kuiper EJ, Klaassen I, Canning P, Stitt AW, Van Bezu J, Schalkwijk CG, Van Noorden CJ, Schlingemann RO. Advanced 
glycation end products cause increased CCN family and extracellular matrix gene expression in the diabetic rodent retina. Diabetologia. 2007;50(5):1089-98.

11. Lee HY, Chung JW, Youn SW, Kim JY, Park KW, Koo BK, Oh BH, Park YB, Chaqour B, Walsh $\mathrm{K}$, et al. Forkhead transcription factor FOXO3a is a negative regulator of angiogenic immediate early gene CYR61, leading to inhibition of vascular smooth muscle cell proliferation and neointimal hyperplasia. Circ Res. 2007;100(3):372-80.

12. Choi JS, Kim KH, Lau LF. The matricellular protein CCN1 promotes mucosal healing in murine colitis through IL-6. Mucosal Immunol. 2015;8(6):1285-96.

13. Komatsu M, Nakamura Y, Maruyama M, Abe K, Watanapokasin R, Kato H. Expression profiles of human CCN genes in patients with osteoarthritis or rheumatoid arthritis. J Orthop Sci. 2015;20(4):708-16.

14. Woo YJ, Seo Y, Kim JJ, Kim JW, Park Y, Yoon JS. Serum CYR61 Is associated with disease activity in Graves' orbitopathy. Ocul Immunol Inflammation. 2018;26(7):1094-100.

15. Zhou F, Zhang Y, Chen D, Su Z, Jin L, Wang L, Hu Z, Ke Z, Song Z. Potential role of Cyr61 induced degeneration of human Muller cells in diabetic retinopathy. PLoS ONE. 2014;9(10):e109418.

16. Matsumae H, Yoshida Y, Ono K, Togi K, Inoue K, Furukawa Y, Nakashima Y, Kojima Y, Nobuyoshi M, Kita T, et al. CCN1 knockdown suppresses neointimal hyperplasia in a rat artery balloon injury model. Arterioscler Thromb Vasc Biol. 2008;28(6):1077-83.

17. Kim I, Park CS. Angiotensin II type 1 receptor blocker, Fimasartan, reduces vascular smooth muscle cell senescence by inhibiting the CYR61 signaling pathway. Korean Circ J. 2019;49(7):615-26.

18. Liu C, Cao Y, He X, Zhang C, Liu J, Zhang L, Wu D, Zhuang X, Xue R, Huang $\mathrm{H}$, et al. Association of Cyr61-cysteine-rich protein 61 and short-term mortality in patients with acute heart failure and coronary heart disease. Biomarkers Med. 2019:13(18):1589-97.

19. Winzap P. Diabetes and baseline glucose are associated with inflammation, left ventricular function and short-and long-term outcome in acute coronary syndromes: role of the novel biomarker Cyr 61. Cardiovasc Diabetol. 2019:18:142.

20. Schober JM, Chen N, Grzeszkiewicz TM, Jovanovic I, Emeson EE, Ugarova TP, Ye RD, Lau LF, Lam SC. Identification of integrin alpha(M)beta(2) as an adhesion receptor on peripheral blood monocytes for Cyr61 (CCN1) and connective tissue growth factor (CCN2): immediate-early gene products expressed in atherosclerotic lesions. Blood. 2002;99(12):4457-65.

21. Shoji M, Takemoto M. Serum podocalyxin levels correlate with carotid intima media thickness, implicating its role as a novel biomarker for atherosclerosis. Sci Rep. 2018;8(1):245.

22. Anand SS, Caron F, Eikelboom JW, Bosch J, Dyal L, Aboyans V, Abola MT, Branch KRH, Keltai K, Bhatt DL, et al. Major adverse limb events and mortality in patients with peripheral artery disease: The COMPASS trial. J Am Coll Cardiol. 2018:71(20):2306-15.

23. Cornejo Del Río V, Mostaza J, Lahoz C, Sánchez-Arroyo V, Sabín C, López S, Patrón P, Fernández-García P, Fernández-Puntero B, Vicent D, et al. Prevalence of peripheral artery disease (PAD) and factors associated: An epidemiological analysis from the population-based Screening PRE-diabetes and type 2 DIAbetes (SPREDIA-2) study. PLoS ONE. 2017;12(10):e0186220.

24. Giovannini S, Tinelli G, Biscetti F, Straface G, Angelini F, Pitocco D, Mucci L, Landolf R, Flex A. Serum high mobility group box-1 and osteoprotegerin levels are associated with peripheral arterial disease and critical limb ischemia in type 2 diabetic subjects. Cardiovasc Diabetol. 2017;16(1):99.
25. He X, Hu X, Ma X, Su H, Ying L, Peng J, Pan X, Bao Y, Zhou J. Elevated serum fibroblast growth factor 23 levels as an indicator of lower extremity atherosclerotic disease in Chinese patients with type 2 diabetes mellitus. Cardiovasc Diabetol. 2017;16(1):77.

26. Zierfuss B, Hobaus C, Herz CT, Pesau G, Koppensteiner R, Schernthaner $\mathrm{GH}$. Thrombospondin-4 increases with the severity of peripheral arterial disease and is associated with diabetes. Heart Vessels. 2020;35(1):52-8.

27. He XX, Su JR, Ma XJ, Lu W, Zhu W, Wang YF, Bao YQ, Zhou J. The association between serum growth differentiation factor 15 levels and lower extremity atherosclerotic disease is independent of body mass index in type 2 diabetes. Cardiovasc Diabetol. 2020;19(1):8.

28. Hayashi A, Shichiri M. Use of noncontact infrared skin thermometer for peripheral arterial disease screening in patients with and without diabetes. Angiology. 2020;71(7):650-7.

29. Biscetti F, Bonadia N, Santini F, Angelini F, Nardella E, Pitocco D, Santoliquido A, Filipponi M, Landolfi R, Flex A. Sortilin levels are associated with peripheral arterial disease in type 2 diabetic subjects. Cardiovasc Diabetol. 2019;18(1):5.

30. Biscetti F, Nardella E, Bonadia N, Angelini F, Pitocco D, Santoliquido A, Filipponi M, Landolfi R, Flex A. Association between plasma omentin-1 levels in type 2 diabetic patients and peripheral artery disease. Cardiovasc Diabetol. 2019;18(1):74

31. Lau LF. CCN1/CYR61: the very model of a modern matricellular protein. Cellular Molecular Life Sci CMLS. 2011;68(19):3149-63.

32. Hviid CV, Erdem JS, Kunke D, Ahmed SM, Kjeldsen SF, Wang YY, Attramadal $\mathrm{H}$, Aasen $\mathrm{AO}$. The matri-cellular proteins "cysteine-rich, angiogenicinducer, 61" and "connective tissue growth factor" are regulated in experimentally-induced sepsis with multiple organ dysfunction. Innate immunity. 2012;18(5):717-26.

33. Hviid CV, Samulin Erdem J, Drechsler S, Weixelbaumer K, Ahmed MS, Attramadal H, Redl H, Bahrami S, Osuchowski MF, Aasen AO. The matricellular "cysteine-rich protein 61 " is released from activated platelets and increased in the circulation during experimentally induced sepsis. Shock (Augusta, Ga). 2014;41(3):233-40.

34. GrzeszkiewiczTM, Lindner V, Chen N, Lam SC, Lau LF. The angiogenic factor cysteine-rich 61 (CYR61, CCN1) supports vascular smooth muscle cell adhesion and stimulates chemotaxis through integrin alpha(6) beta(1) and cell surface heparan sulfate proteoglycans. Endocrinology. 2002;143(4):1441-50.

35. Hilfiker A, Hilfiker-Kleiner D, Fuchs M, Kaminski K, Lichtenberg A, Rothkotter HJ, Schieffer B, Drexler $\mathrm{H}$. Expression of CYR61, an angiogenic immediate early gene, in arteriosclerosis and its regulation by angiotensin II. Circulation. 2002;106(2):254-60.

36. Duan H, He Z, Lin M, Wang Y, Yang F, Mitteer RA, Kim HJ, Yeo E, Han H, Qin $L$ et al. Plasminogen regulates mesenchymal stem cell-mediated tissue repair after ischemia through Cyr61 activation. JCl insight. 2020;5(15).

37. Geovanini GR, Libby P. Atherosclerosis and inflammation: overview and updates. Clin Sci (London). 2018;132(12):1243-52.

\section{Publisher's Note}

Springer Nature remains neutral with regard to jurisdictional claims in published maps and institutional affiliations.

Ready to submit your research? Choose BMC and benefit from:

- fast, convenient online submission

- thorough peer review by experienced researchers in your field

- rapid publication on acceptance

- support for research data, including large and complex data types

- gold Open Access which fosters wider collaboration and increased citations

- maximum visibility for your research: over 100M website views per year

At BMC, research is always in progress.

Learn more biomedcentral.com/submissions 